\title{
UNA PROPUESTA DE ESPECIALIZACIÓN ARCHIVÍSTICA EN 1948. El aGN Y la Escuela de Graduados DE LA UNAM
}

\author{
Ilihutsy Monroy Casillas*
}

\section{Resumen}

El curso de especialización en archivística "Técnica de la investigación histórica en los archivos nacionales" pudo realizarse gracias a la cooperación académica entre el Archivo General de la Nación (AGN), a cargo del licenciado Julio Jiménez Rueda, y la Escuela de Graduados de la Universidad Nacional Autónoma de México (UNAM), dirigida por el doctor José Zozaya. Esta última, un breve experimento educativo que duró diez años -entre 1947 y 1957- tenía por objetivos formar lazos con otras instituciones y aprovechar sus conocimientos e instalaciones para capacitar a los mexicanos. Este texto narra dicha historia a partir del tipo de materias y los maestros que las impartieron, además de la visión que se difundió para considerar sus aportaciones al conocimiento archivístico.

Palabras clave: archivística en México, capacitación archivística, Universidad Nacional Autónoma de México.

\begin{abstract}
The specialization course in archival "Technique of historical research in the National Archives" was possible by the academic cooperation between the Archivo General de la Nación, in charge of Julio Jiménez Rueda, and the Graduate School of the UNAM, directed by Dr. José Zozaya. The latter, a brief educational experiment that lasted ten years -between 1947 and 1957- aimed to develop bonds with other institutions and take advantage of their knowledge and installation to train Mexicans. This text narrates this story, starting from
\end{abstract}

*Archivo Histórico de la UNAM/Instituto de Investigaciones sobre la Universidad y la Educación 
the type of subjects and teachers that taught them, besides the vision that was widespread to consider their contributions to the archival knowledge.

Keywords: Archival in Mexico, archival training, Universidad Nacional Autónoma de México.

Conocer la historia de la enseñanza de la archivística en México tiene un gran sentido práctico y político, ya que nos abre los ojos ante la realidad educativa mexicana y las condiciones históricas de la profesión del archivista. La apuesta por hacer esta historia no es inocente, sino todo lo contrario, porque en el intento de revelar momentos oscuros podemos encontrar elementos para la comprensión integral de los archivos, de la gente que los construye y de la situación general del patrimonio archivístico mismo.

En ese sentido, el relato describe la propuesta de curso de especialización en archivística en 1948, en la cual intervinieron dos instituciones determinantes como el Archivo General de la Nación (AGN) y la Universidad Nacional Autónoma de México (UNAM). Es este artículo un aporte a la historia de la educación que recurre a distintas fuentes documentales y bibliográficas, por lo que funciona como una narración cronológica y análisis de la enseñanza y capacitación archivística mexicana.

\section{El contexto educativo archivístico en los años cuarenta}

Enla más reciente investigación de la historia de la archivística mexicana escrita por Gustavo Villanueva, se nos aclara la construcción del entendimiento archivístico entre 1915 y 1945, a partir de la normativa institucional del AGN y de las siguientes instituciones: la Escuela de Bibliotecarios y Archiveros (1916-1918), la Escuela Nacional de Bibliotecarios (1925) y la Escuela Nacional de Bibliotecarios y Archivistas, la primera ENBA (1945). ${ }^{1}$

Sin duda lo más interesante es la explicación en torno a la cercanía y

1 Villanueva, La archivística, pp. 23-66. En el texto del maestro Villanueva, estos son los casos abordados, pero estoy segura de que hubo otras experiencias de capacitación archivística en el medio educativo en la ciudad y en otras regiones de México, lo cual es tema de otra investigación histórica. 
supeditación entre Biblioteconomía y Archivística. Todo lo que ocurría en la sociedad mexicana de ese entonces lo inclinaba hacia las bibliotecas, debido al fuerte apoyo institucional y económico, al desarrollo y adopción masiva del sistema de clasificación Dewey y a la confusión entre documento de archivo y de biblioteca.

De esta manera, las instituciones que sostenían el título de "archivistas" no solían discutir ni enseñar un método archivístico -porque, además, no existía como tal según lo entendemos en el siglo XXI- ni se hacía honor al Archivo, sino que se perfilaba hacia las bibliotecas.

Por eso, una experiencia de docencia en torno a la archivística en espacios diferentes de la ENBA -donde, por cierto, no se impartió lección alguna de archivística sino hasta 1946, ${ }^{2}$ un año después de su fundaciónllama la atención y se convierte, para este texto, en objeto de investigación.

\section{El AGN}

La larga y compleja historia del AGN -con antecedentes en la época novohispana, y consolidada en 1823-, por sus diversos ámbitos políticos, sociales, económicos, administrativos y culturales tendría un mejor alcance si se hace un análisis de su normativa, ya que ello nos mostraría la evolución de sus políticas con respecto al patrimonio archivístico y al patrimonio histórico-cultural archivístico nacional. Sin embargo, para este texto será suficiente revisar las funciones que se le adjudicaban a partir del Reglamento del Archivo General de la Nación de 1946, vigentes en el momento de la propuesta de convenio con la UNAM.

En el artículo primero, fracción I, el Reglamento señala que el primordial objetivo de la institución era "custodia $[\mathrm{r}]$, clasifica $[\mathrm{r}]$ y cataloga $[\mathrm{r}]$ todos los documentos contenidos en los expedientes [de la Federación]”, para lo cual se organizaría en las siguientes cinco secciones: Paleografía, Investigaciones Históricas, Catalogación, Administrativa y, Biblioteca y Publicaciones.

2 Expedido por el presidente Manuel Ávila Camacho y publicado en el Diario Oficial de la Federación, tomo CLV, número 38, 13 de abril de 1946. Consulté el documento compilado en Mariscal, Reseña histórica, pp. 225-236. Por cierto que este Reglamento estuvo vigente hasta el año de 2012 y 2014, fechas de expedición de la Ley Federal de Archivos y del Reglamento del Archivo General de la Nación, respectivamente. 
Sin embargo, entre sus tareas no figuraba la formación de sus propios recursos humanos, los cuales, caracterizados como técnicos, serían necesarios para el desarrollo de sus tareas y actividades. ${ }^{3}$ Por ejemplo, el Director se debería dedicar, sobre todo, a asuntos político-administrativos (artículos 12-15).

Las secciones de Paleografía, Investigaciones Históricas y Catalogación estarían ejecutando tareas propias de la archivística que modernamente (re) conocemos como identificación, organización (clasificación y ordenación) y descripción; empero, es llamativo cómo se proponen los requisitos para cubrir estas plazas.

Se lee en el artículo 20 que "para desempeñar el puesto de Investigador de Historia, se requiere demostrar capacidad plenamente probada por estudios de especialización realizados en una institución dedicada a la enseñanza de disciplinas históricas o por publicaciones que hayan contribuido al esclarecimiento de hechos históricos". Por su parte, "el jefe de sección de Catalogación deberá ser perito en organización de archivos, con capacidad comprobada por estudios académicos y trabajos realizados en archivos y bibliotecas"; y "para el puesto de catalogador y visitador se necesitará demostrar capacidad plenamente comprobada por estudios de especialización en su ramo en una institución dedicada a la enseñanza de la materia" (artículos 22 y 23). En el caso de los paleógrafos no se especificaba ningún requisito.

Conviene mencionar la opinión del investigador J. Ignacio Rubio Mañé sobre las condiciones del AGN, publicadas en 1940, la cual permite que valoremos aún más esta intención normativa de 1946 por mejorar al Archivo:

Hasta hoy, el propósito de Revilla Gigedo permanece aún irrealizado, a pesar de los largos años transcurridos, porque el Archivo General de la Nación, de la República Mexicana, carece todavía de un edificio adecuado, de un personal integrado por un cuerpo de arcbivistas de escuela y aún no tiene carácter de repositorio

3 A diferencia de lo expresado en el Reglamento del Archivo General y Público de la Nación, de julio de 1916, en el que se señalaba que sus empleados técnicos deberían estar presentes en las conferencias, academias prácticas y lecciones orales sobre paleografía que se darían en el propio establecimiento, y éstos "se tendrán en cuenta de manera especial para el ascenso de los empleados”, según dicta el artículo 23, en Ruiz-Daza, Catálogo documental, p. 142. 
general con tantos otros archivos que se hallan dispersos, algunos abandonados y maltratados, no sólo en la ciudad de México, sino también en los Estados que forman la nación mexicana. ${ }^{4}$

\section{La propuesta de la Escuela de Graduados de la UNAM}

Desde los años treinta la Universidad Nacional pasaba por una crisis económica y una inestabilidad administrativa y política que se extendió hasta la década de los cuarenta. La solución fue la aprobación de una nueva Ley Orgánica en 1945 -aún vigente-, así como la dependencia del subsidio estatal.

El rector interino, doctor Salvador Zubirán, mostró interés en superar las limitaciones económicas a partir de ampliar sus lazos, generando convenios de cooperación con dependencias universitarias y de afiliación con instituciones externas para acercarse a la sociedad mexicana y resolver problemas de manera científica. Todo ello estaría de acuerdo con el artículo primero de la Ley Orgánica, la cual dice que la UNAM “tiene por fin impartir educación superior para formar profesionistas, investigadores, profesores universitarios y técnicos útiles a la sociedad". 5

La mediadora de esta intención educativa y social sería la Escuela de Graduados, aprobada en sesión extraordinaria del Consejo Universitario del 30 de agosto de 1946, la cual estuvo a cargo del doctor José Zozaya, médico muy reconocido. Originalmente, en esta dependencia se concentrarían todas las disciplinas científicas para obtener grados de maestro y doctor, en un momento histórico en el que no estaban delimitadas las diferencias entre grados y títulos -ni siquiera con la recién aprobada Ley Reglamentaria de Profesiones de 1944-, asunto que también llevó a largas confrontaciones al interior de la Universidad. De esta forma, no todos los grados emitidos en la Universidad salieron de esta Escuela. De hecho, su corta duración se explica por la casi nula graduación que tuvo: en diez años sólo hubo diez graduados.

Sin embargo, la experiencia aportada fue muy significativa, pues permitió considerar distintas necesidades disciplinarias, educativas y sociales, además

4 Rubio, El Archivo General, p. 46. Cursivas mías.

5 Monroy, "Una mirada histórica", pp. 73-89. Los siguientes datos sobre la Escuela se extraen de esta investigación. 
constituyó un laboratorio para que la Universidad se conectara con distintas instituciones que estaban a la cabeza en diversas ramas de la investigación científica. Ejemplos gratificantes fueron los relativos a ingeniería sanitaria, enfermería, medicina, música y, desde luego, archivística.

\section{El curso "Técnica de la investigación histórica en los Archivos nacionales"}

Según Gustavo Villanueva, antes de 1940, en la Facultad de Filosofía y Letras de la Universidad Nacional ya se habían impartido cursos relativos a la biblioteconomía - pero no a la archivística-, como las conferencias de 1922 y 1924 a cargo de Juan B. Iguíniz. ${ }^{6}$ La siguiente propuesta vendría hasta 1947, cuando la Escuela de Graduados tuvo a bien relacionarse con el AGN.

Como director del Archivo estaba Julio Jiménez Rueda, reconocido literato, investigador y docente quien publicaría libros como Historia de la literatura mexicana o Herejías y supersticiones de la Nueva España. Interesado en el convenio de afiliación con la UNAM, organizó un curso y lo propuso al doctor Zozaya, primero en septiembre de 1947 y luego en marzo de 1948. ${ }^{8}$ Para realizar los seminarios, Jiménez Rueda fue nombrado por la Universidad como jefe de la División de Humanidades de la Escuela de Graduados, cargo en el que presentó un reglamento y, según los datos del fondo documental de la Escuela de Graduados, este único curso. Si bien en algunos documentos el profesor añadía la posibilidad de ampliar los seminarios en el AGN hacia la temática de la literatura mexicana, esto ya no se llevó a cabo.

6 Villanueva, op. cit., p. 53.

7 "Julio Jiménez Rueda", en Hombres de México, p. 102. Por cierto que el profesor Jiménez Rueda también impartió la materia historia de la cultura universal en la Escuela Nacional de Bibliotecarios y Archivistas, según consta en el oficio del director, licenciado Héctor R. Olea, el 10 de noviembre de 1948, AGN/Archivo de Archivo, Correspondencia particular, Caja 22, Exp [4], f. 14.

8 Oficio de Julio Jiménez Rueda a José Zozaya, 1947, septiembre 29 y 1948, marzo 11, en Instituto de Investigaciones sobre la Universidad y la Educación/Archivo Histórico de la UNAm/Fondo Escuela de Graduados/Posgrado, Caja 17, Exp. 105, f. 5 y 8. En el Archivo General de la Nación/Archivo del Archivo, Correspondencia particular, Caja 22, Exp. [1], se localiza un expediente con documentos sobre el asunto, aunque está incompleto en comparación con el del AHUNAM. A continuación se cita como AHUNAM/EGP. 
El título general del curso era "Técnica de la investigación histórica en los Archivos Nacionales", aunque en una primera versión se llamó tan solo "Técnica de la investigación histórica", y en su versión final comprendería los siguientes cuatro seminarios: el primero, "Historiografía", otro "de Bibliografía", uno más "de Paleografía", y el último llamado "de Organización de Archivos Históricos".

Los profesores encargados de impartir los cursos fueron los licenciados Edmundo O’Gorman y Francisco González de Cossío, y los señores Luis G. Ceballos y Agustín Hernández. Por su importancia en el curso, conviene citar algunos datos biográficos, aunque debe señalarse que, en el caso de dos de ellos se puede acceder fácilmente a su información, tal como adelante se señala.

O’Gorman, aunque formado como abogado, pronto se dedicó a la investigación y reflexión histórica. Su estancia en el AGN, de 1938 a 1952, le permitió acercarse de manera profunda a los documentos históricos, pero también a muchas otras tareas cercanas a las actividades archivísticas. Por ejemplo, realizaba copias certificadas y estudios diplomáticos, y trabajó en la "investigación y clasificación documental", por lo que comentó y publicó cientos de documentos en el Boletín del AGN. ${ }^{9}$ Al mismo tiempo, su pasión por la historia lo llevó a impartir distintas materias sobre la misma en la Facultad de Filosofía y Letras. ${ }^{10}$ Para este curso fue contratado como profesor asociado de historia.

Agustín Hernández Piuki, encargado de impartir la materia de organización de archivos Históricos, estudió en la Escuela de Administración de la Universidad Nacional, y desde joven laboró en archivos (en los del Departamento del Impuesto sobre la Renta de la Secretaría de Hacienda y Crédito Público, Archivo de la Tesorería de la Federación, archivos de la Secretaría de Relaciones Exteriores, así como en el Archivo de la ex Dirección de Pensiones Civiles y Retiros) e impartió materias relativas a los archivos históricos y archivología, sobre todo desde su participación en la fundación de la ENBA en 1945. Cercano colaborador del archivista

9 Galeana, "Edmundo O'Gorman", pp. 14 y 16. Debo indicar que de los expedientes del AGN y del AHUNAM/EGP consultados para esta investigación, no se puede entresacar más información que sean aportaciones valiosas sobre la obra de O'Gorman.

10 Villegas, "El itinerario de un historiador", p. 48. 
Francisco Gamoneda, hizo estudios relativos a los archivos en Estados Unidos y en Cuba, y participó en la Primera Reunión Internacional de Archivos (1961, EUA). Publicó distintos cuadros de clasificación y manuales para la organización de archivos, así como un texto de terminología sobre archivos. ${ }^{11}$ En esta ocasión fue contratado como profesor asociado de archivología.

Las materias del curso fueron descritas de la siguiente forma: "Seminario de Bibliografía [...] Estudio de las bibliografías mexicanas de los siglos XVI, XVII y XVIII, particularmente de esta última para dar a los estudiantes idea de las fuentes en que deben fundar el estudio histórico que van a emprender", impartido por el licenciado Francisco González de Cossío;" "Seminario de Historiografía [...]. Tema general.- La cultura mexicana en el siglo XVIII. Investigación en el Ramo de la Inquisición sobre procesos relativos a la historia de las ideas en ese siglo", dirigido por el licenciado Edmundo O'Gorman; ${ }^{13}$ y "Seminario de Paleografía [...] Estudio de documentos de los siglos dieciséis y diecisiete y dieciocho [sic] en sus diferentes características. Determinación de la autenticidad de estos documentos. Cooperación con la cátedra de [espacio en blanco] para la lectura de los documentos que se necesiten decifrar [sic] en ella", a cargo del profesor Luis G. Ceballos. ${ }^{14}$ Estas tres materias tenían en sus propuestas una especialización histórica en la época novohispana, aunque, en el desarrollo de los seminarios, el de historiografía fue más filosófico -se interesó en hacer una revisión panorámica de la historia como ciencia para llegar a entender la historiología, ${ }^{15}$ y planteó la necesidad de "una historia desde el hombre y no contra el hombre"-, por lo que no cumplió sus objetivos iniciales. En los otros dos cursos se revisaron asuntos técnicos -como las tipografías o la bibliografía religiosa y seglar, la diplomática y revisión de la mitad de los documentos del fondo Hospital de Jesús-y los trabajos finales se propusieron como publicaciones debido a las aportaciones históricas que lograron.

11 Datos biográficos tomados de Villanueva, op. cit., pp. 94-95.

12 AhunAm/EgP, Caja 17, Exp. 105, f. 2, y el informe está en fs. 62-63.

13 Ibid., f. 3, y el informe está en fs. 68-69.

14 Ibid., f. 4, y el informe está en fs. 66-67.

15 Historiología refiere a teoría de la historia, la cual tiene la intención de ubicar patrones y causas en los procesos históricos. 
El seminario Organización de Archivos Históricos, por su parte, contendría los temas de "Manejo y clasificación de los expedientes. Formación de los índices. Problemas que se plantean en la organización de un archivo histórico". Planeado para impartirse en cinco meses, en el "Informe" entregado, sin fecha ni autor, se indica que:

Se desarrollaron ocho temas fundamentales: Introducción a la Archivología, Documentología, Organización, Documental, Trámite, Archivotecnia, Catalotecnia, Expedientología y Archivonomía; analizándose al objeto de la Archivología, definición, origen, objeto y utilidad de los archivos, se hizo una breve reseña histórica de los archivos españoles y mexicanos más importantes; su evolución y los métodos de clasificación del Consulado, el de la Academia de la Historia, el General de la Nación, el de Protocolos, el de Ayuntamientos, el de Arzobispado, el del Museo de Antropología, etc.

Se habló de la técnica del documento, se le estudió como unidad del organismo documental, se analizaron sus características desde su aspecto físico, los elementos que lo forman hasta su denominación, se resaltó la importancia de la organización y se dedicó un capítulo importantísimo a la reconstrucción de documentos.

Después se expusieron las secuelas de trámite, el sistema de registro y control de correspondencia actualmente usado en los archivos públicos, la formación de cuadros clasificadores y los sistemas de organización.

El apartado siguiente estudió los sistemas de catalogación, su redacción, la manera como deben formarse y su división.

Después se consideró el expediente como unidad del Archivo, la secuela de su formación, el ordenamiento de los documentos dentro de los expedientes y la ordenación de ellos en los diferentes sistemas de archivos, los métodos que se usan en los préstamos de expedientes, la transferencia y el sistema de costura de expedientes.

Uno de los capítulos más importantes de la documentología, es la necesidad de unificar y standarizar [sic] los sistemas y procedimientos de trabajo en los archivos.

Se expusieron los planes de organización de los archivos especialmente los históricos, los reglamentos de los archivos, las características necesarias de los 
locales, moblajes y materiales de las dichas instituciones, se propugnó por la reducción física de los archivos.

El último capítulo se dedicó a estudiar las estadísticas de Archivo y la labor indispensable y precisa de la publicidad y divulgación del material que custodian.

Como trabajo final del curso se está formulando una guía de los archivos históricos de la ciudad de México. ${ }^{16}$

El resumen de los temas tratados en este seminario no hace sino despertar muchas inquietudes, -por ejemplo, relativas a la bibliografía y a los sistemas de clasificación decimal usados-, que tristemente no tienen contestación porque no hay más datos al respecto. De estos documentos resaltan varios elementos: si bien el profesor Hernández Piuki se había especializado, en la práctica, en archivos que ahora llamaríamos de trámite y de concentración, en la docencia se centró en los históricos. Y si bien el título limitaba los estudios a los históricos, muchos de los temas abordados en ese semestre se relacionan con los documentos de archivo antes de transferirse a los históricos. Por lo que se lee, él no sólo se centró en aspectos teóricos, sino hasta en asuntos de conservación e instalaciones, por lo que para esos momentos era un curso de archivística integral. En ese sentido, su conocimiento panorámico y actualizado permitió a los asistentes renovarse en estos temas. Es una gran lástima que no contemos con más datos de la discusión sostenida en clase entre asistentes -también archivistas- y el docente especializado, pero, por lo menos, los apuntes mencionados nos dan una idea de lo revisado.

En cuanto a los asuntos escolares, para ser considerado alumno del curso los candidatos debían haber terminado el bachillerato en letras o la maestría, que para el momento sólo había en historia, además de pagar una cuota de inscripción de 50 pesos. ${ }^{17} \mathrm{El}$ ritmo del curso sería el siguiente: una reunión de dos horas y media, dos veces por semana durante un semestre, y el local de reunión sería el propio AGN, con sede en el Palacio Nacional.

Cada seminario implicaría "un crédito de dos unidades", los cuales serían computables para la maestría y el doctorado. Para aprobar los

16 AHUnAm/EgP, Caja 17, Exp. 105, fs. 64-65. 17 Ibid, fs. 9-10. 
seminarios debía cumplirse con cierta asistencia y entregar un trabajo final. No contamos con las listas de asistencia.

Los alumnos inscritos y calificados fueron los siguientes: Guadalupe Pérez San Vicente, Fernando Anaya Monroy, Manuel Salazar Córdoba, la "alumna especial" María González Sánchez y Yolanda Mariel de Ibáñez, aunque esta última no se inscribió en el seminario de Organización de archivos. ${ }^{18}$ Sus calificaciones fueron ochos y dieces. En otra constancia se aseguró que también habían asistido -sin cubrir los requisitos de alumnosocho personas más, entre los que destacan Ernesto de la Torre y Berta Ulloa. ${ }^{19}$ Entre estos asistentes, el personal del propio Archivo, identificado según un listado de 1946, estuvo integrado por los siguientes: Guadalupe Pérez San Vicente y Ernesto de la Torre, los cuales fungían como pasantes de historia en la Sección de Catalogación. ${ }^{20}$

El reducido número de inscritos hizo decir a Jiménez Rueda, en una comunicación a Zozaya del 6 de septiembre, que "no es de esperarse una matrícula copiosa en virtud de que los cursos de esta naturaleza tienen un carácter de seminarios", pero además porque "estos estudios por primera vez se ofrecen a estudiosos de este tipo”. Más adelante advierte que estos cursos "son [...] los primeros que en la división de Humanidades se organizan y me atrevo a pensar que, a pesar de todas las circunstancias adversas el éxito obtenido no es desdeñable". ${ }^{21}$

La defensa que hizo Jiménez Rueda de los cursos por su novedad, y la necesidad de que los grupos fueran pequeños, contrasta con una afirmación realizada en el mismo documento respecto a la nulidad de ingresos por concepto de cuotas: "Las personas que han concurrido a los cursos sin inscripción son en su totalidad empleados del Archivo que pretende[n] adquirir o completar una preparación técnica que los capacite para desempeñar mejor su cometido", los cuales aun con un trabajo remunerado no pueden completar la suscripción. De la misma forma, señaló que los interesados en estos cursos no tenían capacidad para cubrir un pago oneroso, ya que "un aspirante a historiador o maestro en letras es generalmente pobre".

18 Ahunam/EgP, Caja 17, Exp. 105, fs. 42 y 45.

19 Ibid., f. 44.

20 "Lista del personal del Archivo General de la Nación, con expresión de su categoría y sección en que presta sus servicios", en Mariscal, Reseña histórica, pp. 168-169.

21 AHUNAM/EgP, Caja 17, Exp. 105, fs. 46-47. 
El curso, como sería costumbre para la Escuela de Graduados, fue promocionado en distintas instituciones y escuelas, entre otras El Colegio de México, el Instituto Nacional de Antropología e Historia, la Escuela Normal para Maestros, la Biblioteca Benjamín Franklin y, por supuesto, la Escuela de Bibliotecarios y Archivistas. ${ }^{22} \mathrm{Y}$ de esta propaganda académica nos habla una anécdota: la contemporánea asistencia de un grupo de guatemaltecos a los cursos de archivística de la UNAM impartidos por el AGN, aunque en la prensa se mencionó a la ENBA equivocadamente. ${ }^{23}$ Los cursos, tal como se apreciaba, eran pertinentes socialmente.

\section{Conclusiones}

La narración anterior podría llevarnos a suponer que el curso sobre historia y archivos que promovió Julio Jiménez Rueda en el AGN, con el apoyo de la Escuela de Graduados de la UNAM, fue todo un éxito; nos llevaría a considerar que, debido a la excelencia académica de los profesores y a la novedad de los cursos, a la posibilidad de ampliar horizontes históricos y archivísticos, y aun a pesar de no contar con una multitudinaria respuesta, ${ }^{24}$ los seminarios permanecieron por muchos años. Sin embargo, esto no fue así, ya que sólo se dieron durante 1948.

¿Qué fue lo que sucedió? ¿Qué otras condiciones o elementos evitaron la consolidación en el ámbito académico, no sólo del curso de archivística sino de los conocimientos archivísticos? Preguntas éstas que, de manera tangencial, responde la investigación histórica de Gustavo Villanueva, y que, a su vez, piden más asistencia y dedicación por parte de los actuales profesionistas de la archivística.

Causas que definitivamente influyeron en el abandono de este curso por parte de las instituciones fueron el poco interés político, laboral,

22 Oficio de Jiménez Rueda a Francisco Orozco, director de la Escuela de Bibliotecarios y Archivistas, 31 de mayo de 1948, AGN/Archivo de Archivo, Correspondencia particular, Caja 22, Exp. [4], f. 3.

23 "Tres maestros hacen estudios en esta ciudad", en Excélsior, 1948, octubre 5.

$24 \mathrm{Si}$ bien la asistencia fue menor, los cursos permitieron una valiosa preparación de jóvenes como historiadores. Por supuesto me refiero a Berta Ulloa, Ernesto de la Torre y Guadalupe Pérez San Vicente, quienes posteriormente rescataron documentos y acervos como parte importante de su quehacer académico. 
educativo y hasta económico. Otras respuestas posibles son el papel de la biblioteconomía, la cual impidió un desarrollo de la archivística; la integración de la división de Humanidades a la Facultad de Filosofía y Letras de la UNAM, lo que provocó enfrentamientos entre grupos; o la situación del propio AGN. ${ }^{25}$

Finalmente, sería una gran falta no mencionar el posterior intento de la Universidad Nacional por "capacitar y acreditar a los archivistas con la creación en 1956 del Colegio de Biblioteconomía y Archivonomía que en 1966 cambió el nombre a Bibliotecología y Archivología, aunque vivió un posterior cierre en 1974". ${ }^{26}$ Después de eso, en la Universidad sólo se aprecia el apoyo a la archivística a través de la creación de plazas de académicos en el Archivo Histórico de la Universidad Nacional Autónoma de México, ${ }^{27}$ y en cuestiones educativas mediante opciones de técnico en el Colegio de Ciencias y Humanidades; ${ }^{28}$ además, con la reciente creación del programa de la licenciatura en Administración de Archivos y Gestión Documental, que todavía está por ser aprobada en Consejo Universitario. ${ }^{29}$ Muchas historias nos quedan en esa gran laguna temporal, definitivamente.

25 En el Informe final de labores, del doctor Jiménez Rueda, fechado el 14 de abril de 1952, éste dijo que "si el Archivo no cuenta con un personal suficiente, técnico, especializado en historia, paleografía, archivonomía, seguirá constituyendo un serio problema y no podrá prestar servicios eficaces a la investigación de nuestra historia. Hasta ahora un número reducido de empleados, abnegados, honorables, con una sórdida retribución han hecho esfuerzos heroicos para salvar los acervos y dar un servicio eficaz al público; pero esto no basta". Por tanto, la situación de la enseñanza y capacitación archivística no se había mejorado. En Rubio, El Archivo General, p. 62.

26 Villanueva, op. cit., p. 161.

27 Monroy, Una reflexión archivistica, pp. 97-107.

28 Islas, La archivística, p. 152.

29 "Proyecto de creación". 


\section{Fuentes y bibliografía}

Archivo General de la Nación, fondo Archivo del Archivo.

Archivo Histórico de la UNAM/Instituto de Investigaciones sobre la Universidad y la Educación, fondo Escuela de Graduados/Posgrado.

Excélsior.

Hombres de México. Doce mil grandes. Enciclopedia biográfica universal, México, Promexa, 1982.

"Proyecto de creación de la Licenciatura en Administración de Archivos y Gestión Documental", Universidad Nacional Autónoma de MéxicoEscuela Nacional de Estudios Superiores, Unidad Morelia, 2014.

Galeana, Patricia, "Edmundo O'Gorman, arquitecto de la historia", en Edmundo O'Gorman en el Archivo General de la Nación, México, AgN, 1999.

Islas Pérez, María Estela, La archivística en México, México, RenAies/ AHBUAP, 2003.

Mariscal, Mario, Reseña bistórica del Archivo General de la Nación (1550-1946), México, Secretaría de Gobernación, 1946.

Monroy Casillas, Ilihutsy, "Una mirada histórica a la Escuela de Graduados a través de su fondo documental (1946-1957)", en Gustavo Villanueva Bazán, coordinador, Teoría y práctica archivística VI, México, IISUE/UnAm, 2012, pp. 73-89.

"Una reflexión archivística e histórica sobre el concepto del patrimonio documental en México", tesis de maestría en Gestión Documental y Administración de Archivos, Palos de la Frontera, Universidad Internacional de Andalucía, 2012. 
Rubio Mañé, J. Ignacio, El Archivo General de la Nación México, Distrito Federal, Estados Unidos Mexicanos, Conmemoración del sesquicentenario de la fundación del Archivo General de la Nación México 1823-1973, México, Secretaría de Gobernación, 1973.

Ruiz-Daza Cruz, María Elena, Catálogo documental Archivo General de la Nación, 175 aniversario, 23 de agosto de 1823-23 de agosto de 1998, México, AGN, 1998.

Villanueva Bazán, Gustavo, "La archivística: una ciencia en busca de sí misma (México, 1915-1945)", tesis de maestría en gestión documental y administración de archivos, Palos de la Frontera, Universidad Internacional de Andalucía, 2012.

Villegas Moreno, Gloria, "El itinerario de un historiador", en Josefina MacGregor, coordinadora, Homenaje a Edmundo O 'Gorman, México, Facultad de Filosofía y Letras/unam, 2001. 